\title{
The Reliability of Bubble Inclinometer and Tape Measure in Determining Lumbar Spine Range of Motion in Healthy Individuals and Patients
}

\author{
Rahim Sadeghi ${ }^{1}$, Zahra Mosallanezhad ${ }^{*}$, Afsun Nodehi-Moghadam ${ }^{1}$, Mohammad Reza Nourbakhsh ${ }^{2}$, Akbar Biglarian $^{3}$, Kamran Ezati $^{1}$
}

1. Department of Physiotherapy, University of Social Welfare and Rehabilitation Sciences, Tehran, Iran

2. Department of Physical Therapy, University of North Georgia, Georgia, USA.

3. Department of Biostatistics, University of Social Welfare and Rehabilitation Sciences, Tehran, Iran.

4. Department of Physiotherapy, School of Medicine, Guilan University of Medical Sciences, Guilan, Iran.

Cftation: Sadeghi R, Mosallanezhad Z, Nodehi-Moghadam A, Nourbakhsh MR, Ezati K, Biglarian A. The Reliability of Bubble Inclinometer and Tape Measure in Determining Lumbar Spine Range of Motion in Healthy Individuals and Patients. Physical Treatments. 2015; 5(3):137-144.

do

Article info:

Received: 31 Mar. 2015

Accepted: 07 Aug. 2015
Keywords:

Chronic non-specific low back pain, Lumbar range of motion, Reliability, Bubble inclinometer, Tape measure

\section{A B S T RA C T}

Purpose: Regarding the high prevalence of low back pain in various communities and the need to determine an appropriate treatment plan for these patients, examining their functional limitation and disability level is of utmost importance. In this regard, one of the important indicators is Lumbar range of motion. Measurement of the range of motion is a common and appropriate method for determining the functional limitation of the spine and also to examine the effectiveness of various therapeutic interventions. This study was conducted with the purpose of examining the reliability of measuring lumbar range of motion using bubble inclinometer and tape measure.

Methods: This methodological study was performed on 20 healthy males (29-52 years old) and 13 male patients with chronic non-specific low back pain (30-58 years old) in 2015 . The ranges of lumbar forward and backward and side bending were measured with bubble inclinometer and rotation with tape measure for both groups. Two measurements were conducted in one day with an interval of one hour to examine the within day reliability, and a third measurement was conducted one week later to examine the between days reliability. Statistical inference was made through calculation of intraclass correlation coefficients (ICC) and standard error of measurement (SEM). All data analysis was done by SPSS version 18 .

Results: The ICC and SEM values related to the within days and between days reliability were acceptable. The within day and between days ICC range were $0.770-0.982$ and $0.835-0.977$, respectively. SEM range was $0.38-1.20$. However, the results of the reliability values of between days measuring of extension in prone position, by using bubble inclinometer, in patients with slight low back pain were low (ICC $=0.177$ and $\mathrm{SEM}=5.35$ ).

Conclusion: Results of the present study showed that measuring the lumbar range of motion with bubble inclinometer and tape measure (except measuring extension in prone position by using bubble inclinometer in patients with low back pain) was highly reliable. Therefore, these 2 non-invasive and reliable tools can be used to measure the lumbar range of motion and also to follow-up the effectiveness of therapeutic interventions.

\footnotetext{
* Corresponding Author:

Zahra Mosallanezhad, PhD

Address: Department of Physiotherapy, University of Social Walfare and Rhabilitation Sciences, Koodakyar Ave., Daneshjoo Blvd., Evin, Tehran, Iran. Phone: +98 (21) 22180039

E-mail: zmosallanezhad@yahoo.com
} 


\section{Introduction}

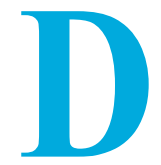

espite the advances in medicine with regard to spinal injuries ailments and treatment methods, lumbar back pain continues to be one of common health problems in industrial and non-industrial countries [1]. More than $84 \%$ of people experience lumbar back pain (LBP) during their lifetime. The incidence of chronic back pain has been $23 \%$, out of which $11 \%$ to $12 \%$ suffer from disability $[2,3]$.

LBP refers to feeling pain between the $12^{\text {th }}$ rib and the inferior gluteal fold with or without pain in leg(s) [24]. Based on etiology, LBP is classified into 2 groups of specific and non-specific LBP. About 10\% of patients suffer from specific LBP and 90\% from non-specific LBP [2, 5]. In non-specific LBP, contrary to the specific one, a specific pathology cannot be found. Therefore, in examining the non-specific LBP a particular factor cannot be named as the cause of the pain. In this type of LBP, the pain usually exacerbates with activity and relatively improves with rest $[2,3]$. Based on the studies conducted in Iran, the incidence of LBP is relatively high like other countries of the world. In Iran, different incidences of LBP have been estimated and reported in different age and occupational groups such as $17 \%$ in school children, $21.8 \%$ in teachers, $62 \%$ in nurses, $84 \%$ in pregnant women, and $84.8 \%$ in surgeons [6-10]. One of the difficulties patients with LBP experiences is limitation of motion in the lumbar spine area, which has direct association with the level of pain $[11,12]$. Because of high incidence of LBP in various communities and the need to determine an accurate treatment plan for patients suffering from such ailment, examining the level of disability and dysfunction is of high importance. In this regard, an important indicator is accurate measurement of the Lumbar range of motion.

Measuring the motion range has been recommended as a common and appropriate method to determine the functional limitation of the spine and also for evaluating the effectiveness of various therapeutic interventions [13-16]. Researchers have developed numerous methods for measuring the Lumbar range of motion. Although, these methods may not be the indicator of the real level of intervertebral motion, they are considered indicators for measuring the lumbar range of motion. Numerous tools and methods are used for measuring the range of motion, including the flexible ruler, tape measure $[17,18]$, spinal mouse, goniometer [19, 20], inclinometer [21], the distance from the fingertip-to-floor $[18,22]$, spinal motion analyzer [23, 24], and radiography [17, 25].
Each of these methods has its own advantages and drawbacks. Among the drawbacks are high cost, exposure to X-ray, the need for special tools and training to use those tools, patient's discomfort, and the lack of proper reliability. For example, according to the report of the American Academy of Surgeons, the coefficient of variation for using the common goniometer had been more than 53\% [26]. In many studies, the inclinometer has been used for measuring the level of bending forward, backwards extension, and side flexion, with different results $[21,27,28]$. Considering the various types of inclinometer, including the mechanical, universal, deluxe, digital, and bubble, in this study, we used the bubble inclinometer because of its relative inexpensiveness, facility of application, accessibility and non-invasiveness.

The present study was designed to determine the reliability of the bubble inclinometer in measuring the lumbar range of motion in the sagittal and frontal planes in healthy individuals and also in patients suffering from non-specific LBP. Given that the basis of bubble inclinometer work is gravity, measuring the lumbar rotation range of motion would not be possible by this tool. Therefore, in this study, for measuring the Lumbar range of motion, the tape measure was used to determine its reliability. This study is part of a clinical trial designed to examine the effect of dry needles (Dry Needling) on reduction of pain, functional disability, and lumbar range of motion in patients suffering from myofascial pain syndrome of quadratus lumborum muscle. Meanwhile, the bubble inclinometer and the tape measure were used as tools to examine the effectiveness of therapeutic intervention.

Because of the importance of measuring the Lumbar range of motion in assessing the disability in patients suffering from lumbar back pain and also in assessing the effectiveness of various therapeutic methods, and considering the lack of general agreement upon this matter, the above study was designed so that the reliability of measuring the lumbar range of motion by bubble inclinometer and the tape measure could be examined by the same examiner.

\section{Materials \& Methods}

This methodological study was conducted for assessing the reliability of measuring the lumbar range of motion by inclinometer in healthy adults and in patients suffering from LBP (20 healthy males and 13 male patients suffering from non-specific LBP) in Ramsar Physiotherapy Clinic, Ramsar, Iran in 2015. Sample size was calculated 
based on previous studies and the subjects were recruited by convenient sampling method. The healthy individuals under study did not have any history of LBP, spinal deformity, neuromuscular disorders, and musculoskeletal or cardiopulmonary diseases. All participants filled out health questionnaires and the consent forms to participate in the study. Table 1 illustrates the Mean number, standard deviation, age, height, weight, and body mass index of the individuals under the study. For conducting the study, the baseline bubble inclinometer (Model 10602 built by Fabrication Enterprise Inc. USA, Figure $1)$, and non-stretchable measuring tape were used.

First, the reference points on the bodies of the individuals under the study were marked by a special marker. The spinous process of the $1^{\text {st }}$ sacral vertebra and the $12^{\text {th }}$ thoracic vertebra were considered as reference points for measuring the range of motion in bending forward and backward. The spinous process of the $12^{\text {th }}$ thoracic vertebra was considered as reference point for measuring the range of motion of lumbar side flexion, and for measuring the lumbar rotation range of motion, the acromion process and the greater trochanter of the opposite side were considered as reference points $[15,29,30]$.

Two bubble inclinometers were used simultaneously for measuring the lumbar range of motion when bending forward (in the standing position), and one bubble inclinometer for measuring the lumbar range of motion for back extension (in the standing and prone position) and side flexion (in the standing position) (Figure 2). The actual ranges of motion of lumbar area when bending forward and backward were calculated by subtracting the number (degree) obtained by the lower inclinometer from the number (degree) obtained by the upper incli-

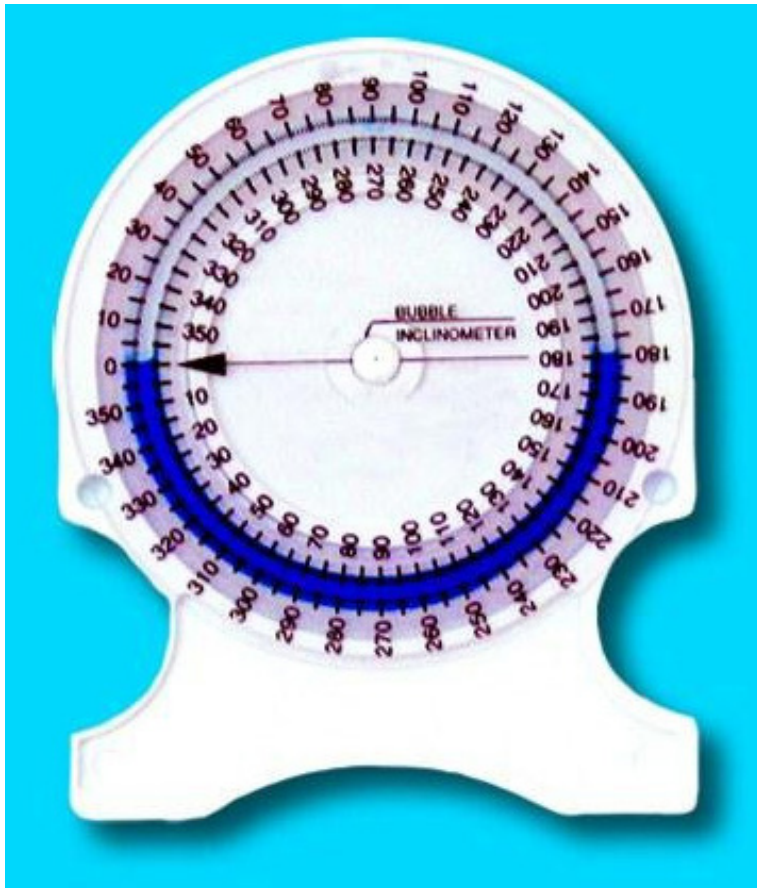

Figure 1. Bubble Inclinometer. $\quad$ PHYSICAL TREA $\$ MENTS

nometer. For measuring the ranges of motion of right and left rotation, the measurement was carried out in the position of sitting on the edge of the bed $[30,31]$. It is to be noted that the platform center of bubble inclinometer was positioned on the reference point and was kept completely in fixed position by the examiner throughout the measurements [29]. The starting point of motion has an intense effect on the measurement, therefore, accurate tactility of reference points is very important $[32,33]$.

Evaluation of the Lumbar range of motion was carried out 3 times and in 2 days. The first and second evaluation
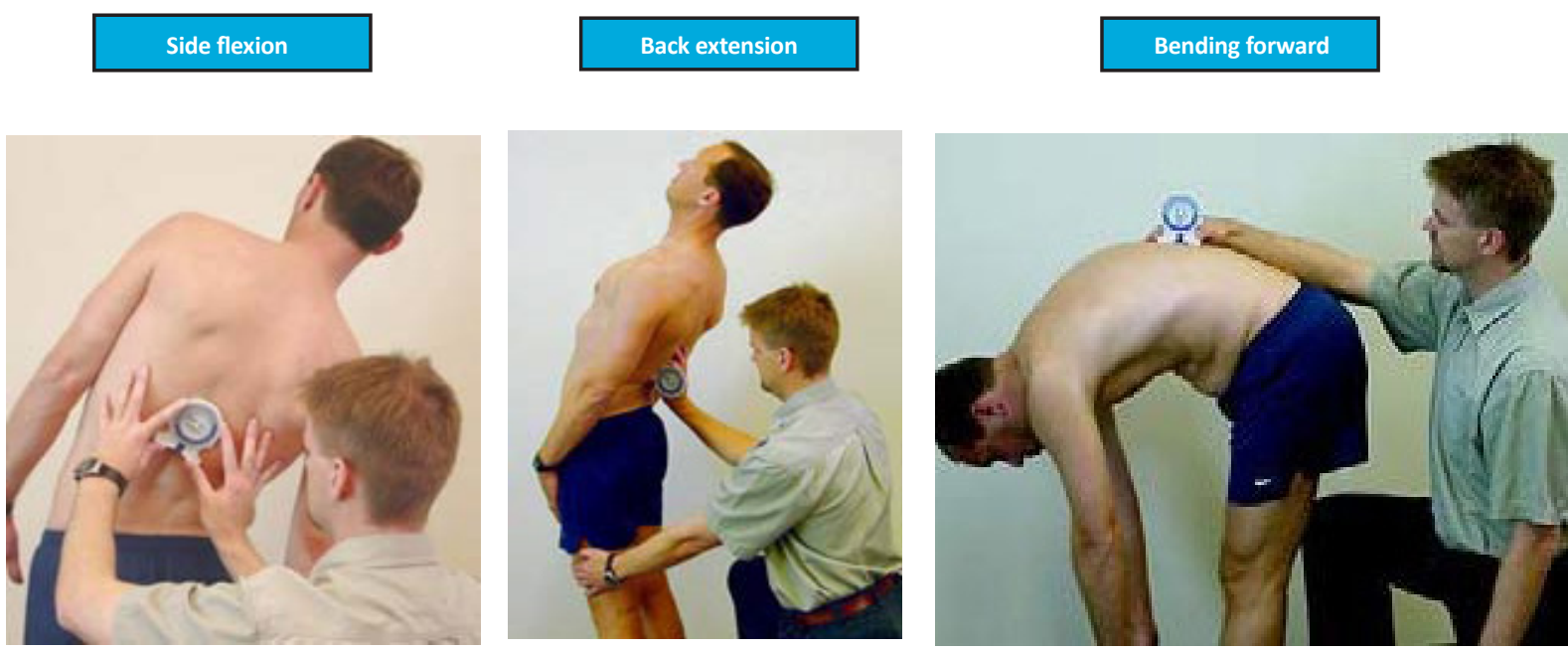

PHYSICAL TREA TMENTS

Figure 2. Method of measuring the lumbar spine range of motion by using bubble inclinometer. 
were carried out in the first day with an interval of 1 hour (for the within day reliability test), and the third examination was carried out a week later (for the between days reliability test). Reliability was based on Rosner classification, i.e. if it is less than $40 \%$, it was considered weak reliability, between $40 \%-75 \%$ was tolerable to good reliability, and more than $75 \%$ was excellent reliability [34]. Data were analyzed using SPSS, version 18, by carrying out the intraclass correlation coefficient (ICC) tests for calculating the relative reliability (reflecting the ability of measurement method to differentiate between the individuals under the study). Also, the standard error of measurement (SEM) was used for evaluating the absolute reliability (reflecting the error in the measurement method).

\section{Results}

The Mean(SD) of age and body mass index of healthy individuals were respectively $40(6.48)$ years and $26.66(2.12) \mathrm{kg} / \mathrm{m}^{2}$, and the median (SD) of age and body mass index of patients suffering from non-specific chronic LBP were respectively $46.85(8.05)$ years and $27.95(2.87) \mathrm{kg} / \mathrm{m}^{2}$ (Table 1). The median and the standard deviation of all measurements of the lumbar range of motion are presented in Table 2. In healthy individuals, the within day and the between days reliability for bending forward were recorded respectively as 0.926 and 0.958 , for back extension respectively as 0.822 and 0.927 , for back extension in the prone position respectively as 0.926 and 0.928 , for the right lateral flexion respectively as 0.895 and 0.896 , for left lateral flexion respectively as 0.935 and 0.946 , for right rotation respectively as 0.770 and 0.835 , and finally for left rotation as
0.823 and 0.853 , respectively (Table 2 ). In patients, the reliability of within day and between days measurement for bending forward were recorded respectively as 0.982 and 0.977 , for back extension in the standing position respectively as 0.952 and 0.964 , for back extension in the prone position respectively as 0.932 and 0.177 , for right lateral flexion respectively as 0.908 and 0.931 , right rotation respectively as 0.937 and 0.971 , and finally for left rotation as 0.944 and 0.958 , respectively (Table 2).

\section{Discussion}

Measuring the spinal range of motion is important in evaluating the spinal function as well as in examining the effect of therapeutic interventions $[21,35]$. In various rehabilitation programs, reliability of evaluation methods is of high importance.

This study aimed to examine the reliability of measuring the lumbar range of motion by using bubble inclinometer and the non-stretchable tape measure. Results of this study showed that the bubble inclinometer has high reliability in measuring the within day and between days range of motion in healthy individuals (ICC $>0.75$ ). According to Richman et al. classification, reliability of the measuring method in the present study is average to high. They considered the ICC measure of $0.80-1.00$ as high, $0.60-0.79$ as average, and $\geq 0.59$ as low [35]. This study also showed that in patients, the bubble inclinometer enjoys high reliability in measuring all of the said ranges of motions in within day test. With regard to between days test, although the reliability of measurement in bending forward, back extension, and side flexions

Table 1. Demographic characteristics of study subjects.

\begin{tabular}{ccccc}
\hline \multicolumn{1}{c}{ Variables } & & Mean & SD & Range \\
\hline \multirow{2}{*}{ Age $(\mathrm{y})$} & Healthy & 40 & 6.48 & $29-52$ \\
& Patients & 46.85 & 8.05 & $30-58$ \\
\hline Height $(\mathrm{cm})$ & Healthy & 173.45 & 6.96 & $162-183$ \\
& Patients & 174 & 6.33 & $64-96$ \\
\hline Weight $(\mathrm{kg})$ & Healthy & 80.35 & 9.43 & $58-98$ \\
\hline & Patients & 84.88 & 11.46 & $20.83-30.3$ \\
\hline
\end{tabular}


Table 2. Results of within day and between days reliability for measuring lumbar range of motion.

\begin{tabular}{|c|c|c|c|c|c|c|}
\hline Variables & & Mean & SD & Within day ICC & Between days ICC & Between days SEM \\
\hline \multirow{2}{*}{$\begin{array}{l}\text { Bending forward } \\
\text { (degree) }\end{array}$} & Healthy & 34.25 & 5.92 & 0.926 & 0.958 & 1.20 \\
\hline & Patients & 25.08 & 7.83 & 0.982 & 0.977 & 1.18 \\
\hline \multirow{2}{*}{$\begin{array}{l}\text { Back extension in } \\
\text { standing position } \\
\text { (degree) }\end{array}$} & Healthy & 7.65 & 2.11 & 0.822 & 0.927 & 0.52 \\
\hline & Patients & 6.92 & 3.07 & 0.952 & 0.964 & 0.60 \\
\hline \multirow{2}{*}{$\begin{array}{l}\text { Back extension in prone } \\
\text { position (degree) }\end{array}$} & Healthy & 5.05 & 1.64 & 0.926 & 0.928 & 0.42 \\
\hline & Patients & 3.23 & 1.42 & 0.932 & 0.177 & 5.35 \\
\hline \multirow{2}{*}{$\begin{array}{l}\text { Right lateral flexion } \\
\text { (degree) }\end{array}$} & Healthy & 18.95 & 2.74 & 0.895 & 0.869 & 0.98 \\
\hline & Patients & 13.46 & 3.6 & 0.908 & 0.931 & 0.97 \\
\hline \multirow{2}{*}{$\begin{array}{l}\text { Left lateral flexion } \\
\text { (degree) }\end{array}$} & Healthy & 18.55 & 3.17 & 0.935 & 0.946 & 0.76 \\
\hline & Patients & 6.92 & 3.77 & 0.937 & 0.971 & 0.63 \\
\hline \multirow{2}{*}{ Right rotation (degree) } & Healthy & 8.2 & 1.28 & 0.770 & 0.835 & 0.56 \\
\hline & Patients & 7.15 & 1.72 & 0.925 & 0.930 & 0.47 \\
\hline \multirow{2}{*}{ Left rotation (degree) } & Healthy & 7.9 & 1.29 & 0.823 & 0.853 & 0.51 \\
\hline & Patients & 7.46 & 1.81 & 0.944 & 0.958 & 0.38 \\
\hline
\end{tabular}

was high in the standing position, in extension while in the prone position the reliability was low $(\mathrm{ICC}=0.177)$.

Examining the reliability of measuring the Lumbar range of motion of right and left rotation by using tape measure showed that in both study groups, the within day test and between days test reliability were excellent. Numerous studies have been reported with regard to reliability of the Lumbar range of motion using various types of inclinometers and in different positions [31]. In discussion sections of the conducted studies, knowledge of various types of inclinometers, measurement time intervals, position of the patient prior and during measurement, and the reference points are important [31]. In the study by Merrit and colleagues, the reliability of measurement of the modified Schober and Moll tests, the distance between "fingertip-to-floor" test and Loebl inclinometer, were examined by 2 examiners on 50 patients with the reference point situated $15 \mathrm{~cm}$ above the spinous procession of the first sacral vertebra. In this study, the reliability of measuring back extension in the standing position (Loebl extension test) by Loebl inclinometer was reported as weak. Also, examining the reliability of forward bending and back extension in the prone position has not been carried out by Loebl in- clinometer. The contradiction of this study results with our study may be due to the type of inclinometer used, study participants, employing 2 examiners, and different reference points [36]. Dillard and colleagues, similar to the study by Mayer and colleagues, used one type of inclinometer. Contrary to the present study, measurement took place by 2 examiners with one week interval. In this study, all measurements were conducted in the standing position. Result of this research was in contradiction to the present study and the researchers of the study believed that fat tissue interfered in determining the anatomical locations [37]. Mellin and colleagues by examining the reliability of the inclinometer method for measuring the range of motion of the back and lumbar area of 27 healthy individuals in bending forward, back extension, and side flexion did not find much difference in different positions. However, these researchers considering the obtained results and facility of work, recommended the sitting position for measuring the range of motion in bending forward, prone position while the hands are placed behind the head for measuring the back extension, and the standing position facing the wall and close to it for measuring the side flexion [32]. 
In the present study, the reliability of measuring the Lumbar range of motion in back extension in the prone position, was determined in healthy individuals as well as patients, and reliability of measurement was different between these groups. The study by Batti'e and colleagues showed that the spine range of motion is affected by age, gender, and physical characteristics [38]. Most studies reported same results regarding the reliability of measuring the Lumbar range of motion when bending forward in the standing position by using different inclinometers and methods. However, with regard to the reliability of measuring the Lumbar range of motion in back extension while in the standing position, their results were different. The reason that some of these results contradict with our study results may be due to the measuring method, sample size, lack of homogeneity of samples, and failure to employ by experienced people. For confirming the results of the present study, it is necessary that similar studies be conducted with longer intervals between the measurements in larger sample sizes and with more accurate variety of inclinometers.

Our limitations in this study included small sample size, not including healthy or sick females, short interval between each measurement, and lack of comparing the results with other measurement methods such as radiology. It is recommended that in future studies, the selection of samples be carried out through the random sampling method and a greater number of individuals be allocated to each group. In the previous studies, the validity of measuring the Lumbar range of motion with the inclinometer was compared with radiology measurements, (and a high correlation was found between these 2 methods), therefore, the inclinometer has been recommended as a reliable tool to assess the lumbar range of motion $[15,39]$. New studies by examining the reliability of measuring methods for lumbar range of motion through utilizing mobile phone applications and comparing its results with those obtained through clinical tools such as bubble inclinometer have reported that iPhone applications are valid, reliable, appropriate, and acceptable [40].

On the whole, results of this study demonstrate that bubble inclinometer and non-stretchable tape measure possess high reliability for measuring the Lumbar range of motion in healthy individuals and in patients with LBP (except in back extension while in the prone position) and they can be used for evaluating the Lumbar range of motion in healthy individuals and in patients suffering from lumbar back pain and also for therapeutic interventions follow up.

\section{Acknowledgments}

We hereby express our thanks and appreciation to the Dean of Ramsar School of Nursing and Midwifery, Dr Shamsalinia. We also appreciate Dr. Zahra Fotokian, member of Faculty of Ramsar School of Nursing and Midwifery and Dr. Ibrahim Sadeghi, member of Faculty of Shomal University in Amol, Iran, and to all study participants for their cooperation. We also acknowledge the University of Social Welfare and Rehabilitation Sciences for the financial support of this research.

\section{Conflict of Interest}

The authors declared no conflict of interests.

\section{References}

[1] Ehrlich GE. Low back pain. Bulletin of World Health Organization. 2003; 81(9):671-76.

[2] Nitsure PV, Pathania TS, Bilgi TA. Comparison of elastic resistance band exercises and yoga in physiotherapy students with chronic non-specific low back pain: a randomized clinical trial. Journal of Yoga \& Physical Therapy. 2014; 5:180. doi: 10.4172/2157-7595.1000180

[3] Balagué F, Mannion AF, Pellisé F, Cedraschi C. Non-specific low back pain. Lancet. 2012; 379(9814):482-91.

[4] Krismer M, van Tulder M. Low back pain group of the bone and joint health strategies for europe project. Best Practice \& Research Clinical Rheumatology. 2007; 21(1):77-91.

[5] Balagué F, Mannion AF, Pellisé F, Cedraschi C. Non-specific low back pain. Lancet. 2011; 379(9814):482-91. doi: 10.1016/ S0140-6736(11)60610-7.

[6] Mohseni-Bandpei MA, Ahmad-Shirvani M, Golbabaei N, Behtash H, Shahinfar Z, Fernández-de-las-Peñas C. Prevalence and risk factors associated with low back pain in Iranian surgeons. Journal of Manipulative and Physiological Therapeutics. 2011; 34(6):362-70.

[7] Mohseni-Bandpei MA, Bagheri-Nesami M, Shayesteh-Azar M. Nonspecific low back pain in 5000 Iranian school-age children. Journal of Pediatric Orthopaedics. 2007; 27(2):12629.

[8] Mohseni-Bandpei MA, Ehsani F, Behtash H, Ghanipour M. Occupational low back pain in primary and high school teachers: prevalence and associated factors. Journal of Manipulative and Physiological Therapeutics. 2014; 37(9):70208.

[9] Mohseni-Bandpei MA, Fakhri M, Bargheri-Nesami M, Ahmad-Shirvani M, Khalilian AR, Shayesteh-Azar M. Occupational back pain in Iranian nurses: an epidemiological study. British Journal of Nursing. 2006; 15(17):914-17. 
[10] Mohseni-Bandpei MA, Fakhri M, Ahmad-Shirvani M, Bagheri-Nessami M, Khalilian AR, Shayesteh-Azar M, et al. Low back pain in 1,100 Iranian pregnant women: prevalence and risk factors. Spine Journal. 2009; 9(10):795-801.

[11] Ferreira ML, Ferreira PH, Latimer J, Herbert R, Maher CG. Does spinal manipulative therapy help people with chronic low back pain? Australian Journal of Physiotherapy. 2002; 48(4):277-84.

[12] Gomez TT. Symmetry of lumbar rotation and lateral flexion range of motion and isometric strength in subjects with and without low back pain. Journal of Orthopaedic \& Sports Physical Therapy. 1994; 19(1):42-48.

[13] Waddell G. Evaluation of results in lumbar spine surgery: clinical outcome measures-assessment of severity. Acta Orthopaedica Scandinavica. 1993; 64(251):134-37.

[14] Hoiriis KT, Pfleger B, McDuffie FC, Cotsonis G, Elsangak $\mathrm{O}$, Hinson $\mathrm{R}$, et al. A randomized clinical trial comparing chiropractic adjustments to muscle relaxants for subacute low back pain. Journal of Manipulative and Physiological Therapeutics. 2004; 27(6):388-98.

[15] Mayer TG, Tencer AF, Kristofferson S, Mooney V. Use of noninvasive techniques for quantification of spinal rangeof-motion in normal subjects and chronic low-back dysfunction patients. Spine. 1984; 9(6):588-95.

[16] Moll J, Wright V. Measurement of spinal movement: the lumbar spine and back pain. New York: Grune \& Stratton; 1976.

[17] Tousignant M, Poulin L, Marchand S, Viau A, Place C. The Modified-Modified Schober Test for range of motion assessment of lumbar flexion in patients with low back pain: a study of criterion validity, intra-and inter-rater reliability and minimum metrically detectable change. Disability and Rehabilitation. 2005; 27(10):553-59.

[18] Gauvin MG, Riddle DL, Rothstein JM. Reliability of clinical measurements of forward bending using the modified fingertip-to-floor method. Physical Therapy. 1990; 70(7):44347.

[19] Fitzgerald GK, Wynveen KJ, Rheault W, Rothschild B. Objective assessment with establishment of normal values for lumbar spinal range of motion. Physical Therapy. 1983; 63(11):1776-781.

[20] Klein AB, Snyder-Mackler L, Roy SH, DeLuca CJ. Comparison of spinal mobility and isometric trunk extensor forces with electromyographic spectral analysis in identifying low back pain. Physical Therapy. 1991; 71(6):445-54.

[21] Burdett RG, Brown KE, Fall MP. Reliability and validity of four instruments for measuring lumbar spine and pelvic positions. Physical Therapy. 1986; 66(5):677-84.

[22] Haywood KL, Garratt AM, Jordan K, Dziedzic K, Dawes PT. Spinal mobility in ankylosing spondylitis: reliability, validity and responsiveness. Rheumatology. 2004; 43(6):750-57.

[23] Troke M, Moore AP. The development of a new form of instrument fixation for the OSI CA 6000 spine motion analyzer. Manual Therapy. 1995; 1(1):43-46.

[24] Troke M, Moore AP, Maillardet FJ, Hough A, Cheek E. A new, comprehensive normative database of lumbar spine ranges of motion. Clinical Rehabilitation. 2001; 15(4):371-79.
[25] Macrae IF, Wright V. Measurement of back movement. Annals of the Rheumatic Diseases. 1969; 28(6):584-89.

[26] Dopf CA, Mandel SS, Geiger DF, Mayer PJ. Analysis of spine motion variability using a computerized goniometer compared to physical examination a prospective clinical study. Spine. 1994; 19(5):586-95.

[27] Williams R, Binkley J, Bloch R, Goldsmith CH, Minuk T. Reliability of the modified-modified Schöber and double inclinometer methods for measuring lumbar flexion and extension. Physical Therapy. 1993; 73(1):26-37.

[28] Nitschke JE, Nattrass CL, Disler PB, Chou MJ, Ooi KT. Reliability of the American medical association guides' model for measuring spinal range of motion: its implication for whole-person impairment rating. Spine. 1999; 24(3):262-68.

[29] Loebl WY. Measurement of spinal posture and range of spinal movement. Rheumatology. 1967; 9(3):103-10.

[30] Magee DJ. Orthopedic physical assessment. 4th ed. Philadelphia: Saunders Publication; 2006.

[31] Saur PM, Ensink FB, Frese K, Seeger D, Hildebrandt J. Lumbar range of motion: reliability and validity of the inclinometer technique in the clinical measurement of trunk flexibility. Spine. 1996; 21(11):1332-338.

[32] Mellin G, Kiiski R, Weckström A. Effects of subject position on measurements of flexion, extension, and lateral flexion of the spine. Spine. 1991; 16(9):1108-110.

[33] Panzer DM. The reliability of lumbar motion palpation. Journal of Manipulative and Physiological Therapeutics. 1992; 15(8):518-24.

[34] Rosner B. Fundamental of biostatics. 1st ed. Belmont: Thomson Brooks; 2006.

[35] Richman J, Makrides L, Prince B. Research methodology and applied statistics, part 3: measurement procedures in research. Physiotherapy Canada. 1980; 32(4):253-7.

[36] Merrit JL, McLean TJ, Erickson RP, Offord KP. Measurement of trunk flexibility in normal subjects: reproducibility of three clinical methods. Mayo Clinic Procedure. 1986; 61(3):192-97.

[37] Dillard J, Trafimow J, Andersson GBJ, Cronin K. Motion of the lumbar spine: reliability of two measurement techniques. Spine. 1991; 16(3):321-24.

[38] Battié MC, Bigos SJ, Sheehy A, Wortley MD. Spinal flexibility and individual factors that influence it. Physical Therapy. 1987; 67(5):653-58.

[39] Portek I, Pearcy MJ, Reader GP, Mowat AG. Correlation between radiographic and clinical measurement of lumbar spine movement. Rheumatology. 1983; 22(4):197-205.

[40] Kolber MJ, Pizzini M, Robinson A, Yanez D, Hanney WJ. The reliability and concurrent validity of measurements used to quantify lumbar spine mobility: an analysis of an iphone application and gravity based inclinometry. International Journal of Sports Physical Therapy. 2013; 8(2):12937. 
